\title{
RR Lyrae studies with Kepler: showcasing RR Lyr
}

\author{
Katrien Kolenberg ${ }^{1,2}$ Robert L. Kurucz $^{1}$, Robert Stellingwerf ${ }^{3}$, \\ James M. Nemec ${ }^{4}$, Paweł Moskalik ${ }^{5}$, Luca Fossati ${ }^{6}$, \\ and Thomas G. Barnes ${ }^{7}$
}

${ }^{1}$ Harvard-Smithsonian Center for Astrophysics, 60 Garden Street, Cambridge MA 02138, USA email: kkolenberg@cfa.harvard.edu

${ }^{2}$ Institute of Astronomy, Leuven University, Celestijnenlaan 200d, 3001 Heverlee, Belgium

${ }^{3}$ Stellingwerf Consulting, 11033 Mathis Mountain Rd SE Huntsville AL 35803-2813, USA

${ }^{4}$ Dept. of Physics \& Astronomy, Camosun College, Victoria, Br. Columbia, V8P 5J2, Canada

${ }^{5}$ Copernicus Astronomical Centre, ul.Bartycka 18, 00-716, Warsaw, Poland

${ }^{6}$ Argelander-Inst. für Astronomie der Univ. Bonn, Auf dem Hügel 71, D-53121, Bonn, Germany

${ }^{7}$ The University of Texas at Austin, McDonald Observatory, $82 \mathrm{Mt}$. Locke Rd.

McDonald Observatory, Texas, 79734, USA

\begin{abstract}
Four years into the Kepler mission, an updated review on the results for RR Lyrae stars is in order. More than 50 RR Lyrae stars in the Kepler field are observed with Kepler and each one of them can provide us with new insight into this class of pulsating stars. Groundbased spectroscopy of the Kepler targets allows us to narrow down their physical parameters. Previously, we already reported a $50 \%$ occurrence rate of modulation in the RRab stars, a large variety of modulation behavior, period doubling in several Blazhko stars, the detection of higherovertone radial modes, probable non-radial modes and new types of multiple-mode RR Lyrae pulsators, among both the RRab and the RRc stars. In addition, the quasi-continuous photometry obtained over several years with Kepler allows one to observe changes in Blazhko behavior and additional longer cycles. These observations have sparked new theoretical modelling efforts. In this short paper we showcase RR Lyr itself. The star has been observed with Kepler in short cadence, and some remarkable features of its pulsation behavior are unveiled in this long-studied prototype, through the Kepler photometry and additional spectroscopic data.
\end{abstract}

Keywords. stars: variables: RR Lyrae, stars: individual: RR Lyr, techniques: photometric, techniques: spectroscopic

\section{Introduction}

Kepler had been operational at full capacity for four years until the second of its four reaction wheels failed in May 2013, thereby compromising its capacity to point accurately. Towards the end of Kepler's nominal mission time, over 40 RR Lyrae stars were known in the observed 115-square-degree field and all of them were included in the target list for the extended mission, which started in November 2012. More recently, several RR Lyrae stars have been found serendipitously, e.g., through the citizen science program "PlanetHunters" (www.planethunters.org), bringing the total of known Kepler RR Lyrae stars to about 50.

To complement the wide-band filter observations of the Kepler targets, Jeon et al. (these proceedings) have obtained extensive multicolor photometry for all the known RR Lyrae stars in the Kepler field. In addition, high-resolution spectroscopy has been obtained for $41 \mathrm{RR}$ Lyrae stars in the Kepler field, leading to an accurate $[\mathrm{Fe} / \mathrm{H}]$ determination for each one (Nemec et al. 2013). An overview of published studies (reviews excluded) on the 
Table 1. RR Lyrae studies with Kepler, and spin-off studies/results published thus far.

\begin{tabular}{|c|c|c|c|}
\hline Topic & Authors & $\mathbf{N}_{\text {stars }}$ & | Remark \\
\hline First results (period doubling) & Kolenberg et al. (2010a) & 2 & A \\
\hline Period doubling in Kepler data & Szabó et al. (2010) & $3+4$ & $\mathrm{~A}, \mathrm{M}$ \\
\hline Flavors of variability & Benkő et al. (2010) & 29 & \\
\hline Mathematical description of modulation & Benkö et al. (2011) & & $\mathrm{M}, \mathrm{S}$ \\
\hline RR Lyr long-cadence data & Kolenberg et al. (2011) & 1 & \\
\hline Testing Stothers model on RR Lyr & Smolec et al. (2011) & 1 & $\mathrm{~A}, \mathrm{M}$ \\
\hline Non-modulated RR Lyrae stars & Nemec et al. (2011) & 19 & \\
\hline Modeling of period doubling & Kolláth et al. (2011) & & $\mathrm{M}$ \\
\hline Radial resonance Blazhko model & Buchler \& Kolláth (2011) & & $\mathrm{M}, \mathrm{S}$ \\
\hline RRc multiperiodicity & Moskalik et al. (2012) & 4 & \\
\hline Nonlinear asteroseismology for RR Lyr & Molnár et al. (2012) & 1 & $\mathrm{~A}, \mathrm{M}$ \\
\hline Multiperiodicity in V445 Lyr & Guggenberger et al. (2012) & 1 & \\
\hline (Quasi-)periodic modulation in BL Her stars & Smolec \& Moskalik (2012) & & M, S \\
\hline Stitching together Kepler data of a Blazhko Star & Çelik et al. (2012) & 1 (example) & $\mathrm{A}$ \\
\hline Comparison of non-repetitive Blazhko cycles & Guggenberger (2012) & 1 & A \\
\hline Metallicity $[\mathrm{Fe} / \mathrm{H}], v_{\mathrm{rad}}$, etc. from spectra & Nemec et al. (2013) & 41 & $\mathrm{~A}, \mathrm{G}$ \\
\hline RR Lyr in short cadence & Stellingwerf et al. (2013) & 1 & \\
\hline Additional RR Lyrae stars? & Kinemuchi (2013) & TBD & $\mathrm{A}, \mathrm{G}$ \\
\hline Chaotic pulsation models & Plachy et al. (2013) & & $\mathrm{M}, \mathrm{S}$ \\
\hline Shock Blazhko model & Gillet (2013) & 1 (example) & M \\
\hline Ground-based multicolor data & Jeon et al. (these proc.) & 41 & G \\
\hline Nonradial modes or not? & Benkő (these proc.) & 41 & A \\
\hline Interchange of alternating ampl & Plachy et al. (these proc.) & 3 & $\mathrm{~A}$ \\
\hline Multi-mode resonances in $R R$ Lyrae st & Molnár et al. (these proc.) & 4 & A, M \\
\hline
\end{tabular}

${ }^{a}$ A: Analysis, M: Modelling, G: Ground-based data, S: Spin-off.

Kepler data and their spin-offs is given in Table 1. In this paper we focus on the brightest RR Lyrae star in the Kepler field, RR Lyr.

\section{Showcasing RR Lyr}

The analysis of the long-cadence Kepler data of RR Lyr (quarters Q1-Q2, May September 2009) was published by Kolenberg et al. (2011). As of Q5 (March 2010) the star was observed in short cadence. Molnár et al. (2012) found evidence for the first radial overtone in the Q5 and Q6 short-cadence data. A frequency analysis performed on the Q5-Q15 short-cadence data (March 2010-January 2013) leads to the following conclusions (see also Stellingwerf et al. 2013):

- There is clear evidence for a longer cycle in RR Lyr. A cycle of duration of about 4 years was reported by Detre \& Szeidl (1973). The Blazhko amplitude variation of about $40 \%$ at the beginning of the Kepler measurements decreases significantly (to as low as 0-10\% in light amplitude) and then increases again (see Fig. 1).

- There is a variation of the 0.5667-day pulsation period (from 0.5655 to 0.5685 day) within each Blazhko cycle, with the shorter period corresponding to the larger amplitude phases in the Blazhko cycle. This variation of half a percent is huge considering that the mean period has barely changed since the observations by Shapley (1916).

- There is a variation of the Blazhko period itself (from 39.2 days at maximum Blazhko amplitude to 38.4 days at the amplitude minimum) over the 4-year modulation cycle, i.e., about $2 \%$.

- There is a small but detectable variation (a few mmag) of the mean brightness over the Blazhko cycle.

Spectroscopic data allow an in-depth analysis of the pulsations of RR Lyrae stars (see also Guggenberger, these proceedings). A spectroscopic data set of RR Lyr, obtained with the high-resolution $(R=60000)$ spectrograph attached to the $2.7-\mathrm{m}$ telescope at McDonald Observatory was described by Kolenberg et al. (2010b). Integration times were 


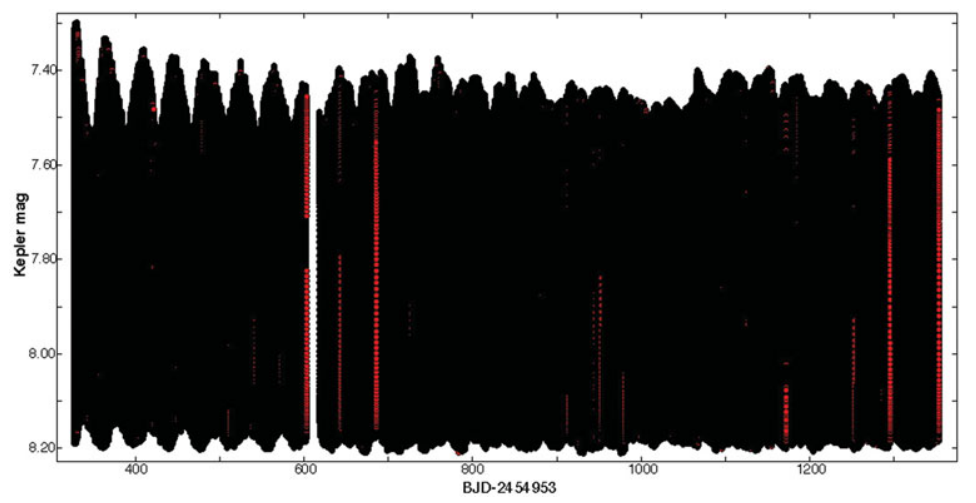

Figure 1. Kepler short-cadence data of RR Lyr (Q5-Q15). Individual cycles are not visible, but the envelope of the Blazhko modulation is. There is clear evidence for a variation in the strength of the Blazhko effect.

typically 20 minutes. A detailed abundance analysis was performed on the spectrum in the most quiescent phase in RR Lyr's pulsation cycle. This is the phase when the star reaches its maximum radius, around pulsation phase $\sim 0.3$ for RR Lyr.

We fitted a static Kurucz atmosphere model to the spectrum in the most quiescent phase. Using an instrumental profile of $5 \mathrm{~km} \mathrm{~s}^{-1}$ (corresponding to the instrumental resolution), the best fit is obtained for a macroturbulent velocity $v_{\text {mac }}=5 \pm 2 \mathrm{~km} \mathrm{~s}^{-1}$ and a projected rotational velocity $v \sin i=5 \pm 2 \mathrm{~km} \mathrm{~s}^{-1}$.

Short-cadence spectroscopy was obtained simultaneously with the short cadence $K e$ pler data over three nights in 2010 (Kolenberg et al., in preparation). With a 1-minute spectrum taken every few minutes the photospheric layers can be followed in detail over time. In Fig. 2 we show the radial-velocity variations for two spectral lines, the $\mathrm{H} \gamma$ line at $4340.462 \AA$ and a Cr line at $4351.811 \AA$, over a few hours of spectra coinciding with the descending branch (declining light) of the star (pulsation phase [0.0-0.35]). We can detect shifts of $1 \mathrm{~km} \mathrm{~s}^{-1}$ for spectra taken only four minutes apart, even in this "quieter" phase interval of the pulsation cycle. As a consequence, spectra taken over 20 minutes, an integration time necessary to obtain a sufficient signal-to-noise ratio for a detailed line profile analysis on a $3-\mathrm{m}$ class telescope, show smearing of the order of $5 \mathrm{~km} \mathrm{~s}^{-1}$. That

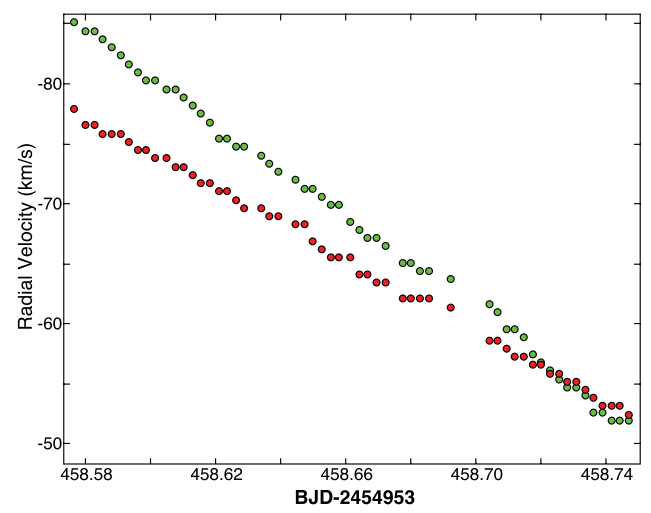

Figure 2. Radial velocities derived from two lines for the short-cadence spectra. The steeper radial-velocity curve corresponds to the $\mathrm{H} \gamma$ line, the other one to the $\mathrm{Cr}$ line. 
implies that the macroturbulent velocity parameter $v_{\mathrm{mac}}=5 \mathrm{~km} \mathrm{~s}^{-1}$ adopted in our fit to the observed spectrum is probably a representative lower limit.

Assuming $v \sin i=5 \mathrm{~km} \mathrm{~s}^{-1}$ implies, for a star with a radius of about $5 R_{\odot}$, that $P_{\text {rot }} \sim 50 \mathrm{~d} \times \sin i$. As a consequence, rotation period and Blazhko period could correspond, which is an essential component in some models for the Blazhko effect (Shibahashi \& Takata 1995; Dziembowski \& Mizerski 2004), though currently not the most favored explanation. The observation of changing or multiple modulation periods does pose a problem for the models linking the modulation directly to the rotation, but one could think of a scenario in which features at different latitudes on the star rotate at different speeds, translated into the light variation. However, often the observed modulation periods for a given star are sometimes too far apart (e.g., 40 days and 120 days) to make this a plausible scenario.

\section{Acknowledgements}

We are particularly grateful to Wojtek Dziembowski for his important contributions to the longstanding puzzle of the Blazhko effect in RR Lyrae stars. KK also is very indebted to him for his encouragement and enthusiasm. KK acknowledges support from Marie Curie Fellowship 255267 SAS-RRL within the 7th European Community Framework Program. Some of her work on the Kepler data was funded through research from the Austrian Research Foundation (FWF projects P19962 and T359).

\section{References}

Benkő, J., Kolenberg, K., Szabó, R., et al. 2010, MNRAS, 409, 1585

Benkő, J, Szabó, R. \& Paparó, M. 2011, MNRAS, 417, 974

Buchler, J. R. \& Kolláth, Z. 2011, ApJ, 731, 24

Çelik, L., Ekmekçi, F., Nemec, J., et al. 2012, arXiv: 1202.3607

Detre, L. \& Szeidl, B. 1973, IBVS, 764

Dziembowski, W. A. \& Mizerski, T. 2004, AcA, 54, 363

Gillet, D. 2013, $A \mathscr{E} A$, 554, A46

Guggenberger, E. 2012, AN, 333, 1044

Guggenberger, E., Kolenberg, K., Nemec, J. M., et al. 2012, MNRAS 424, 649

Kinemuchi, K., 2013, arXiv: 1310.0544

Kolenberg, K., Szabó, R., Kurtz, D. W. et al., 2010a, ApJ, 713, L198

Kolenberg, K., Fossati, L., Shulyak, D., et al., 2010b, A\&\&A 519, 64

Kolenberg, K., Bryson, S., Szabó, R., et al., 2011, MNRAS 411, 878

Kolláth, Z., Molnár, L., \& Szabó, R., 2011, MNRAS 414, 1111

Molnár, L., Kolláth, Z., Szabó, R., et al., 2012, ApJ, 757, L13

Moskalik, P., Smolec, R., Kolenberg, K., et al., 2012, arXiv: 1208.4251

Nemec, J. M., Smolec, R., Benkő, J. M., et al., 2011, MNRAS, 417, 1022

Nemec, J. M., Cohen, J. G., Ripepi, V., et al., 2013, ApJ, 773, 181

Plachy, E., Kolláth, Z., \& Molnár, L., 2013, MNRAS 433, 3590

Shibahashi, H. \& Takata, M., 1995, ASP-CS, 83, 42

Smolec, R. \& Moskalik, P., 2012, MNRAS 426, 108

Smolec, R., Moskalik, P., Kolenberg, K., et al., 2011, MNRAS, 414, 2950

Stellingwerf, R. F., Nemec, J. M., \& Moskalik, P., 2013, arXiv: 1310.0543

Szabó, R., Kolláth, Z., Molnár, L., et al., 2010, MNRAS, 409, 1244

Szeidl, B., 1988, in: G. Kovács, L. Szabados, \& B. Szeidl (eds.), Multimode Stellar Pulsations (Budapest: Konkoly Observatory, Kultura), p. 45 